\title{
Brillouin light scattering under one-dimensional confinement: Symmetry and interference self-canceling
}

\author{
B. Graczykowski, ${ }^{1,2, *}$ A. Gueddida, ${ }^{3}$ B. Djafari-Rouhani, ${ }^{3}$ H.-J. Butt, ${ }^{2}$ and G. Fytas ${ }^{2}$ \\ ${ }^{1}$ Faculty of Physics, Adam Mickiewicz University, Umultowska 85, 61-614 Poznan, Poland \\ ${ }^{2}$ Max Planck Institute for Polymer Research, Ackermannweg 10, D-55128, Mainz, Germany \\ ${ }^{3}$ Institut d'Electronique, de Microélectronique et de Nanotechnologie (IEMN), UMR-CNRS 8520, \\ Département de Physique, Université de Lille 1, F-59655, Villeneuve d'Ascq, France
}

(Received 3 January 2019; published 29 April 2019)

\begin{abstract}
We present the spontaneous Brillouin light scattering (BLS) under simultaneous one-dimensional confinement of sound and light and show that the photon-phonon coupling results from nontrivial interplay of the photoelastic and moving-interface effects. We reveal two types of BLS self-canceling: governed by mode symmetry and driven by destructive interference of the two effects. We show that the latter can be adjusted by the light polarization and phonon wave number. Furthermore, we present a measurement of the shear-horizontal waves in thin membranes.
\end{abstract}

DOI: 10.1103/PhysRevB.99.165431

\section{INTRODUCTION}

Almost one century after the discovery of Brillouin light scattering (BLS) [1] it is hard to overestimate its significance in the research of $\mathrm{GHz}$ phonons. Being well established in the nondestructive elastic evaluation [2,3], BLS is nowadays under continuous development focused on phononic crystals [4-7], bioimagining [8], or magnonics [9,10]. The physical origin of BLS has significant implications in the fields of optomecahnics [11,12], stimulated BLS in fiber optics [13], or coherent light sources [14]. Spontaneous BLS originates from inelastic light scattering on thermally populated acoustic waves/phonons, where light couples to sound through fluctuation of the dielectric function. It results either from the photoelastic (PE) effect or the dynamic surface corrugation known as the surface-ripple (SR) effect. In principle, the BLS spectrum reflects the mechanical and optical properties of the material, scattering geometry, selection rules, and possible interference of the two aforementioned scattering effects. Notably, the latter becomes essential upon spatial confinement to dimensions commensurate the wavelength of the probing light [15-17]. For the first time, the interplay of PE and SR effects was observed for surface acoustic waves using spontaneous BLS [15]. A few decades later, the advent of cavity optomechanics $[11,12]$ and stimulated BLS in waveguides and microfibers $[13,18-21]$ has brought a surge of interest, in fact, in the same interplay phenomenon. Here, the perturbation of the dielectric constant due to surface vibrations has a more general description and is referred to as the moving-interface (MI) effect $[22,23]$. In this context, submicrometer membranes are simple and model structures offer simultaneous confinement and coupling of hypersonic phonons and photons in the visible range. The past decade has faced a flourishing of fundamental research on thin membranes focused on their applications

*Corresponding author: bartlomiej.graczykowski@amu.edu.pl in photonics, optomechanics, phononics, flexible electronics, and heat transport management [24-27]. Among these, BLS proved the conversion of nondispersive bulk waves into hypersonic dispersive Lamb waves propagating in thin metallic [28,29], semiconducting [30,31], dielectric [32], or polymer membranes [33]. However, to date the focus was mostly on the spectral position of particular acoustic modes, i.e., the dispersion relation and elastic properties under confinement. At the same time the BLS intensity, despite being well explained for supported thin films [16,34], remained unexplored or misinterpreted. In the following we will investigate this forgotten spectral parameter and show how 1D confinement of $\mathrm{Si}$ leads to features of BLS not encountered in bulk or supported thin films. In particular, we present two mechanisms of BLS self-canceling governed by the acoustic mode symmetry or the interference of PE and MI effects. To gain comprehensive understanding and explain the experimental data we develop a finite element method (FEM) model based on the classical elasto- and electrodynamics.

\section{MATERIALS AND METHODS}

\section{A. Brillouin light scattering experiment}

We use single-crystal $\mathrm{Si}$ membranes to investigate the simultaneous 1D spatial confinement of photons and phonons and its implications in BLS. The membrane is free standing without any mechanical or optical coupling with a supporting substrate. Its surface is coplanar to (001) and the edge is defined along [110] of Si. We intentionally use $d=260 \mathrm{~nm}$ thick samples since they remain semitransparent for the used laser light $\left(\lambda_{0}=532 \mathrm{~nm}\right)$ and work as an optical cavity. In addition, the chosen thickness is comparable to the probed acoustic waves. Therefore, the first few orders of Lamb waves can be observed simultaneously. We performed BLS measurements in the transmission $(\theta \mathrm{A})$ geometry schemed in Fig. 1(a), which has not been applied to thin membranes, 


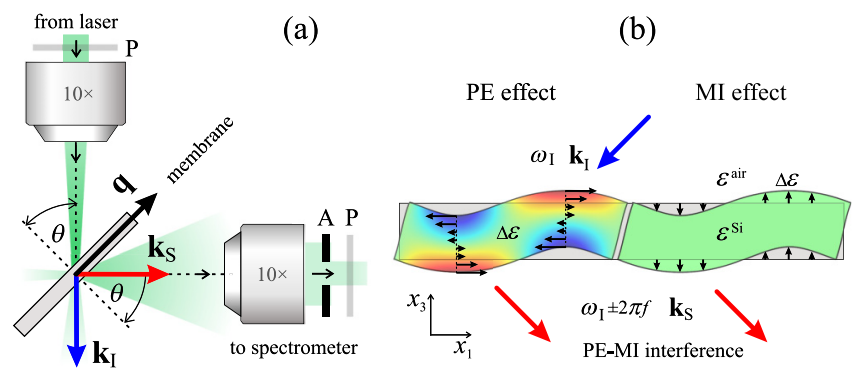

FIG. 1. (a) Scheme of the angle resolved BLS in the transmission geometry applied to a membrane. Symbols A and P denote aperture and polarizer, respectively. (b) Schematic presentation of photoelastic (PE) and moving-interface (MI) effects resulting from the flexural acoustic wave propagating in the membrane. The local change of the dielectric constant $\Delta \varepsilon$ is depicted by a color scale and horizontal arrows for PE effect and vertical arrows for MI effect. $2 \theta$ is the scattering angle while symbols $\mathbf{k}_{\mathrm{S}}, \mathbf{k}_{\mathrm{I}}$, and $\mathbf{q}$ stand for acoustic, scattered light, and incident light wave vectors, respectively.

so far. We used $p p$, ss, and $s p$ configurations of the incident and scattered light, where $p$ denotes light polarized parallel to the sagittal plane (TM polarization) and $s$ normal to it (TE polarization) [2]. BLS probes phonons with wave vector $\mathbf{q}$ which is geometrically selected by the momentum transfer $\pm \mathbf{q}=\mathbf{k}_{\mathrm{S}}-\mathbf{k}_{\mathrm{I}}$ with $\mathbf{k}_{\mathrm{S}}$ and $\mathbf{k}_{\mathrm{I}}$ being the wave vector of the scattered and incident photons, respectively (Fig. 1). In the $\theta \mathrm{A}$ geometry and for the PE effect, $\mathbf{q}$ lies in the plane of the membrane and is of magnitude $q=4 \pi \sin \theta / \lambda_{0}$, where $2 \theta$ is the scattering angle. For the MI effect we assume that the momentum conservation, similarly as in the SR mechanism, holds for the in-plane components of $\mathbf{k}_{\mathrm{S}}$ and $\mathbf{k}_{\mathrm{I}}$. Hence $q$ is identical to that of the PE effect.

\section{B. Finite element method model}

Figure 1(b) shows schematically photoelastic (PE) and moving-interface (MI) effects resulting from the deformation of a membrane corresponding to flexural acoustic wave. The PE effect is a bulk phenomenon, resulting from the local strain induced by the acoustic wave. As a consequence the dielectric constant $\varepsilon_{i j}(i, j=1,2,3)$ of the material undergoes a local variation $\delta \varepsilon_{i j}(\mathbf{r})=p_{i j k l}(\mathbf{r}) u_{k l}(\mathbf{r})$, where $p_{i j k l}$ denotes components of the PE tensor, $u_{k l}$ are elements of the strain tensor, and $\mathbf{r}$ is the position vector. The MI effect results from the variation of $\varepsilon_{i j}$ in close vicinity of each interface due to the acoustic motion. This contribution which is proportional to the amplitude of vibration at the interface can be written as

$$
\delta \varepsilon_{i i}=\left(\varepsilon^{\mathrm{Si}}-\varepsilon^{\mathrm{air}}\right)\left[u_{3} \delta\left(x_{3}+2 / d\right)-u_{3} \delta\left(x_{3}-d / 2\right)\right],
$$

where $\varepsilon^{\mathrm{Si}}$ and $\varepsilon^{\text {air }}$ are dielectric permittivities of Si and air, respectively, and $u_{3}$ is the displacement along the $x_{3}$ axis. The delta functions show the localization of the MI effect at the upper and lower interfaces of the membrane. Then, a general expression for the scattered electric field $\mathbf{E}^{\mathrm{S}}$ can be calculated by using a Green function approach [35-37] or a simulation technique based on the FEM model [38] as used in this work. Our FEM model resembles the experimental conditions including the material properties [39-41] and scattering geometry [Fig. 1(b) and Fig. S1 in the Supplemental Material
[42]]. To calculate the efficiency of the Brillouin scattering, we follow a simulation method in which, for a given phonon mode, we evaluate the modulation of the scattered light by the acoustic vibration. More precisely, we calculate the scattered optical field at successive instants of an acoustic period under the assumption that the acoustic mode strain profile is being frozen at these instants. This hypothesis is justified by the fact that the frequency of the phonon is several orders of magnitude lower than the optical frequency. The calculation of the scattering from the incident light is based on the following four steps: (i) phonon dispersion curves and their eigenvectors, (ii) transmission and reflection of the incident light, (iii) different orders of scattering, and (iv) efficiency of BLS. Each of these steps is explained in detail in Sec. S1 in the Supplemental Material [42] . However, in order to discriminate the modes which are BLS active based on their symmetry, we use a simple expression for scattering intensity $I_{\mathrm{PE}}[43,44]$. Here, we assume that the electromagnetic field is traveling in a homogeneous background (without a dielectric contrast). In this case the only mechanism contributing to the scattering is the PE effect:

$$
I_{\mathrm{PE}}=\left|E_{j}^{\mathrm{S}}\right|^{2} \propto\left|\frac{1}{2 \pi f} \int \exp (i \mathbf{q} \cdot \mathbf{r}) p_{i j k l} u_{k l} E_{i}^{\mathrm{I}} d V\right|^{2},
$$

where $E_{j}^{\mathrm{S}}$ and $E_{i}^{\mathrm{I}}$ are $i$ th and $j$ th components of the scattered and incident electric fields, respectively, $f$ and $\mathbf{q}$ are frequency and wave vector of the acoustic wave, and the $V$ denotes the probed volume.

\section{RESULTS AND DISCUSSION}

Figure 2 presents a comparison of the measured (a)-(c) and calculated (d)-(f) BLS spectra at different $\theta$ in the form of a dispersion relation $f(q)$ for three different polarizations. The color scale refers to the intensity of the scattered light, while the solid, dashed, and dotted lines indicate the calculated dispersion of antisymmetric (A), symmetric (S), and shear-horizontal ( $\mathrm{SH})$ acoustic waves, respectively. At first glance, we can notice that the calculations match well both the experimental dispersions and intensities of the modes. The former agreement supports the assumption for $q(\theta)$ being the same for PE and MI effects. As follows from Figs. 2(a)-2(c), A modes remain silent for all polarizations while $S$ modes are present in $p p$ and $s s$. The dispersion obtained for the $s p$ polarization reveals only the fundamental SH0 mode.

We start the discussion with $\mathrm{SH}$ and A modes, which are silent in the experimental dispersions plotted in Figs. 2(a)-2(c). Although this behavior is well reproduced by the FEM data [Figs. 2(d)-2(f)] it can be qualitatively explained by means of Eqs. (1) and (2) adapted to the scattering geometry (Sec. S4 in the Supplemental Material [42]). For any SH mode the out-of-plane displacement $\left(u_{3}\right)$ is zero and according to Eq. (1) the MI effect is BLS inactive. Hence, for the $s p$ polarization, the presence of $\mathrm{SH} 0$ as well as the absence of higher order SH modes in Figs. 2(c) and 2(f) are solely due to the PE effect. Furthermore, as follows from Eq. (2), the PE effect can result from nonzero components of the strain tensor $u_{i j}$ which do not vanish after the integration over membrane thickness $\left(x_{3}\right)$. In other words, the PE effect self cancels if 


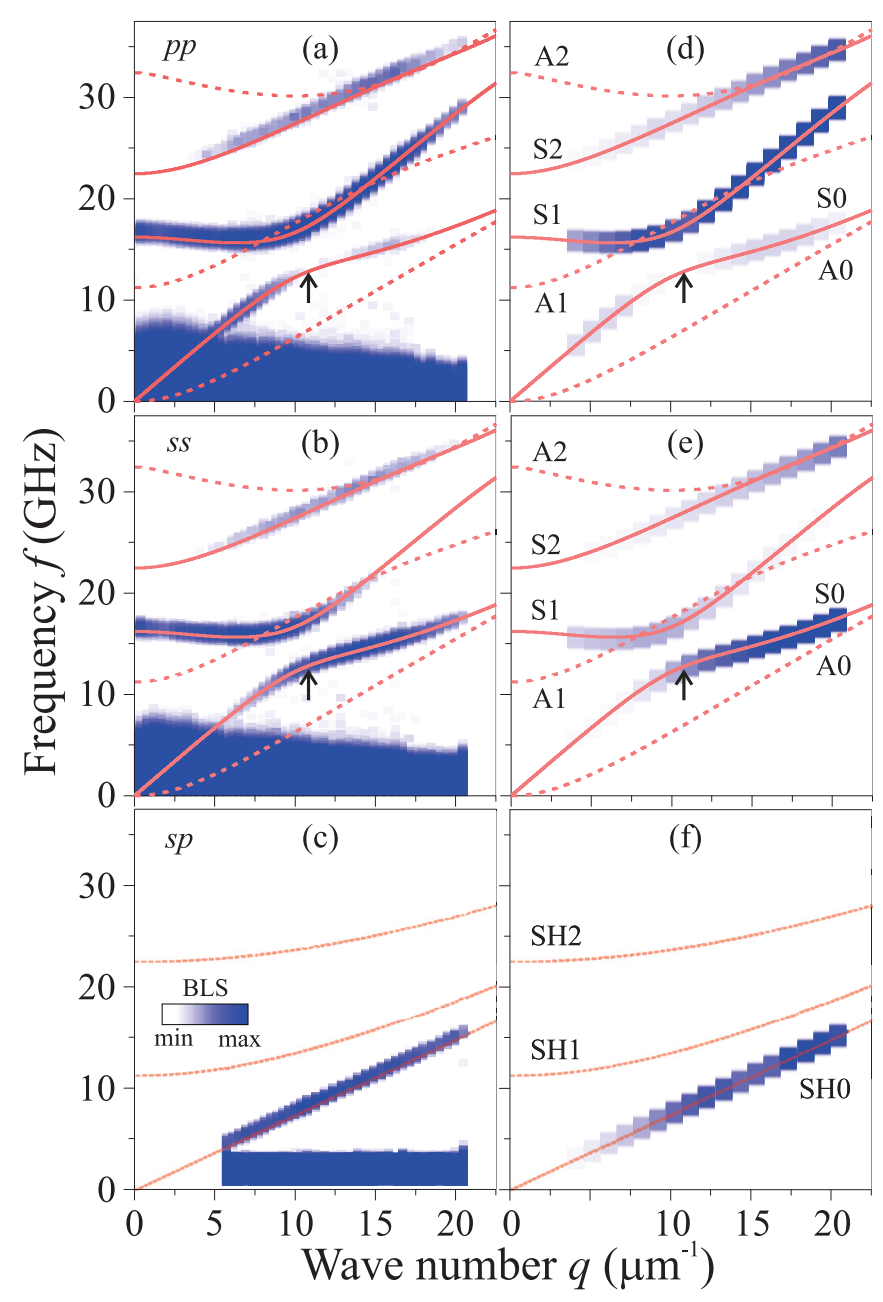

FIG. 2. BLS (a)-(c) and FEM (d)-(f) dispersion relation maps for the $\mathrm{Si}$ membrane obtained from the transmission $(\theta \mathrm{A})$ BLS geometry at the three configurations of incident and scattered light $(p p, s s$, and $s p$ ). Symbols $\mathrm{A}, \mathrm{S}$, and $\mathrm{SH}$ refer to antisymmetric, symmetric, and shear-horizontal acoustic Lamb waves, respectively. Arrows refer to an exemplary spectrum discussed in the text.

the corresponding $u_{i j}$ is antisymmetric with respect to the membrane midplane. To answer whether PE vanishes due to this symmetry condition, we use FEM $2 \mathrm{D}$ maps ( $x_{1} x_{3}$ plane) of the strain tensor components plotted in Fig. 3. For the first three SH modes displayed in Fig. 3(a) we can conclude that SH0 mode remains BLS active. This is in agreement with experimental and numerical results in Figs. 1(c) and 1(f). As follows from Fig. 3(a), SH1 mode should be BLS active due to the symmetry of $u_{23}$. Nevertheless, the PE effect for SH1 is several orders of magnitude weaker than for SH0 mode (Fig. S3 in the Supplemental Material [42]). Further, the higher odd harmonics are suppressed by the thermal population factor $f^{-2}$. Notably, the PE effect associated with SH2 mode is self-canceled as 2D maps of $u_{12}$ and $u_{23}$ reveal their antisymmetry. The same scenario applies to higher order even SH modes so that they are not present in the dispersions in Figs. 1(c) and 1(f). For $p p$ and ss polarizations, the PE effect can result from nonzero components of $u_{i j}$, which are plotted in Fig. 3(b) for exemplary A0 and S0 modes. For A0 (a)

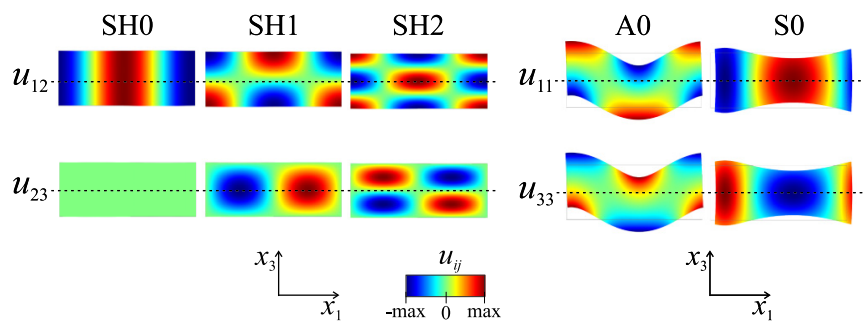

FIG. 3. FEM 2D maps displaying the symmetry and antisymmetry of the strain tensor $u_{i j}$ components corresponding to (a) first three orders of SH waves and (b) fundamental A0 and S0 waves. Dashed lines indicate midplaned of unperturbed membranes. Calculations were performed for a single wavelength wide unit cell and at $q=$ $6.28 \mu \mathrm{m}^{-1}$. The deformation of the unit cell is associated with the displacement $u_{i}$. The omitted maps are of $u_{i j}=0$ due to $u_{1}=u_{3}=0$ for $\mathrm{SH}$ and $u_{2}=0$ for $\mathrm{A}$ and $\mathrm{S}$ modes.

mode both $u_{11}$ and $u_{33}$ are antisymmetric and consequently the PE effect is self-canceled. The same situation applies to all higher order A modes. Albeit, for A0 mode $u_{3} \neq 0$; thus BLS activity should be warranted by the MI effect. However, the out-of-plane displacement of any A mode does not modulate the membrane thickness and according to Eq. (1) the MI effect from two interfaces is self-canceled. Overall, the missing modes in Fig. 2 are a consequence of the individual selfcanceling PE and MI effects due to the membrane midplane symmetry of SH and A modes.

For the BLS active $\mathrm{S}$ modes the intensities in Figs. 2(a) and 2(b) depend on their order/branch and the wave number. Notably, this dependence is different for $p p$ and $s s$ polarizations, what is correctly reproduced by FEM calculations in Figs. 2(d) and 2(e) as well. Referring to S0 mode in Fig. 3(b) we notice that $u_{11}$ and $u_{33}$ are symmetric with respect to the membrane midplane, and thereby the PE effect is not self-canceled. For this mode, the membrane thickness is modulated so that the MI effect can contribute to BLS in addition to the PE effect. To understand the complex behavior of $\mathrm{S}$ modes we analyze the interplay of the PE and MI effects for exemplary BLS spectra at $q=12 \mu \mathrm{m}^{-1}$ (arrows in Fig. 2). In Figs. 4(a) and 4(b) we compare BLS spectra measured in $p p$ and $s s$ polarizations, respectively. The corresponding FEM results reproduce well the experimental spectra and provide individual contributions of PE and MI effects to BLS. A brief comparison of the calculated decomposed and resulting spectra points to the nontrivial superposition of PE and MI effects for $p p$ polarization, whereas the MI effect dominates the BLS signal in the ss polarization. The interplay of PE and MI effects is clearly manifested in the case of S0 mode in the $p p$ polarization. Namely, superposition of significant contributions of PE and MI effects drastically reduces the BLS intensity of this mode. This behavior can be explained by the destructive interference of the light scattered due to PE and MI effects (Fig. S2 in the Supplemental Material [42]). Overall, the BLS activity of S modes is both light polarization and phase-dependent superposition of the PE and MI effects which lead to interference induced self-canceling of BLS. 


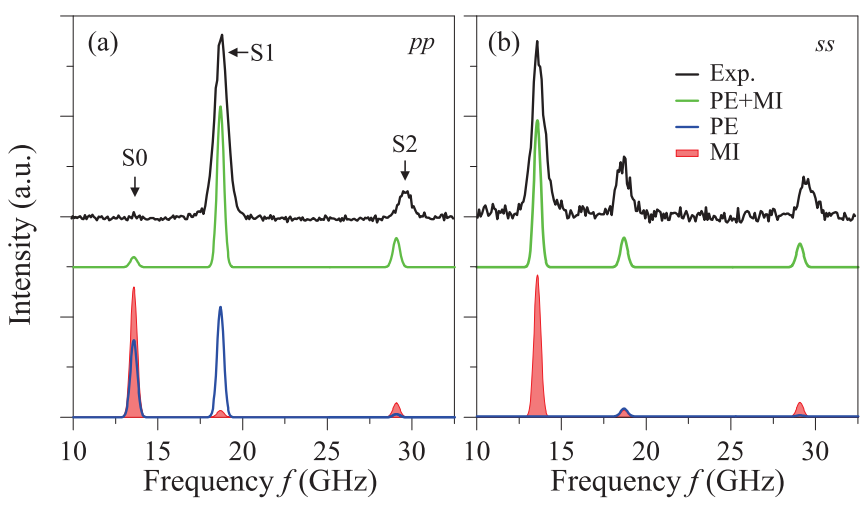

FIG. 4. Experimental (black line) and calculated (green line) BLS spectra (anti-Stokes) at $q=12 \mu \mathrm{m}^{-1}$ in (a) $p p$ and (b) $s s$ polarization. Spectra are decomposed into the individual contribution of PE (blue line) and MI (red shading) effects. The calculated spectra are broadened by the instrumental linewidth of $0.2 \mathrm{GHz}$.

The presented description of the BLS in thin membranes can be extended to any other experimental configuration. Here, we consider the example of the backscattering (BS) geometry $[2,42]$. To date, in this configuration BLS from thin membranes has been solely assigned to the SR effect [30-33,45]. This was supported both by small scattering volume and behavior of $q(\theta)$ matching the SR not the PE effect. To revise this assumption, we use the dispersions obtained for $p p$ and $s p$ polarizations (Fig. 5). A comparison with the corresponding data in Fig. 2 obtained from the $\theta$ A geometry reveals similarity and an additional branch of $\mathrm{A} 0$ mode in the $p p$ polarization. Furthermore, $\mathrm{SHO}$ mode is again active in the $s p$ polarization although in the case of cubic symmetry materials there is no PE coupling for shear waves in the BS geometry [2]. In this case SH0 mode is BLS active due to the reflection of the incident light from the back surface of the membrane [inset to Fig. 5(d)]. The reflected beam works as a secondary incident beam $\mathbf{k}_{\mathrm{I}}^{\prime}$ which together with the scattered light $\mathbf{k}_{\mathrm{S}}^{\prime}$ resemble the $\theta$ A geometry with $q(\theta)$ being the same as for the SR effect. Hence the PE effect can contribute and further interfere with the MI effect for $p p$ and $s s$ polarizations in the $\mathrm{BS}$ in the same manner as in the $\theta \mathrm{A}$ configuration. On the other hand, the activation of $\mathrm{A} 0$ mode in the $p p$ BS geometry is due to the incomplete compensation of the MI effects from two surfaces of the membrane. Here, the MI effect from the back side of the membrane is reduced by a factor proportional to the attenuation of light traveling twice between two sides of the sample (Fig. S5 in the Supplemental Material [42]).

\section{CONCLUSIONS}

In summary, we presented experimental evidence and accounted for the self-canceling of spontaneous BLS under 1D confinement of silicon. We showed that this phenomenon can

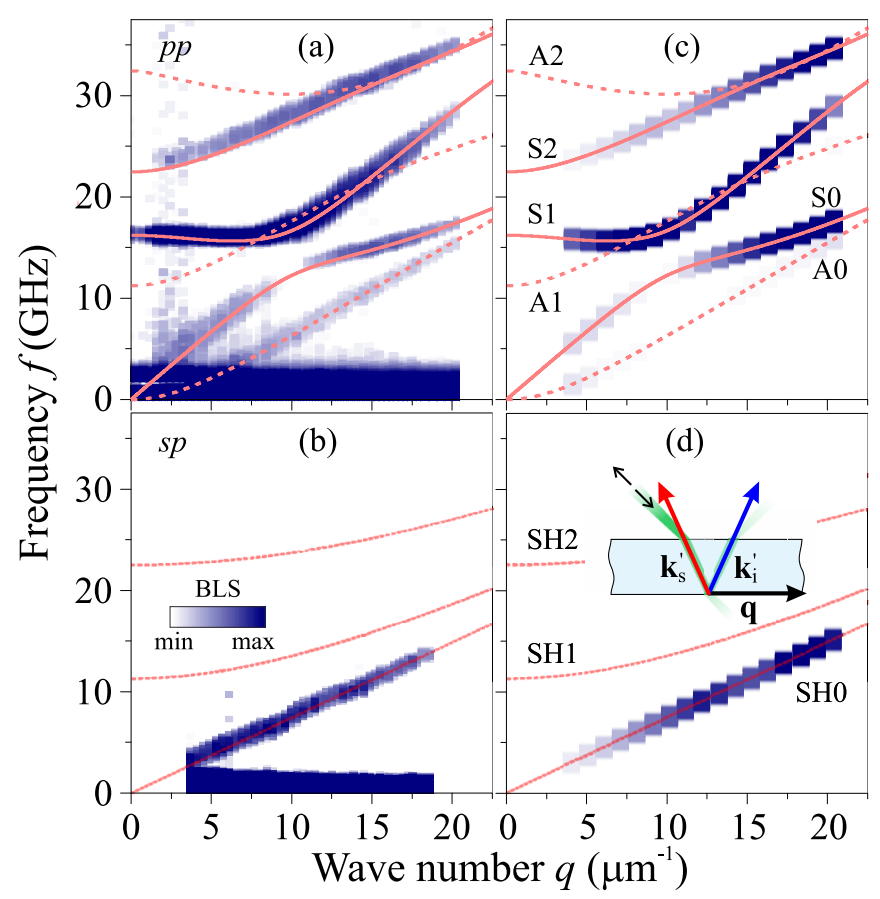

FIG. 5. BLS (a),(b) and FEM (c),(d) dispersion relation maps for the Si membrane obtained from the backscattering (BS) geometry at two configurations of incident and scattered light polarization ( $p p$ and $s p$ ). The inset to (d) refers to a secondary incident beam created by the light reflected from the back side of the membrane.

result from two distinct mechanisms: individual canceling of the PE and MI due to symmetry of the confined acoustic modes and the destructive interference of the PE and MI effects. The latter mechanism depends on wavelength, symmetry, and order of the acoustic mode, and, furthermore, on the direction and polarization of light. In contrast to previous studies, we provide a more general description of BLS in thin membranes including both photoelastic and moving-interface effects and their nontrivial coupling. In addition, we reported an experimental observation of shear-horizontal modes in submicrometer membranes. Our results demonstrate geometrical degrees of freedom for the PE and MI coupling which are easily adjustable from the material exterior: light polarization and acoustic wavelength. In perspective this approach can be extended to more complex structures and provide a platform for tailorable photon-phonon interaction with implications in phononics, stimulated BLS, and optomechanics.

\section{ACKNOWLEDGMENTS}

B.G. acknowledges the support from the Homing (Homing/2016-1/2) and First Team (POIR.04.04.00-005D1B/18-00) programs granted by the Foundation for Polish Science. B.D.-R. acknowledges the support from the FET OPEN H2020 project PHENOMEN (Grant No. 713450). The work was supported by the ERC AdG SmartPhon (Grant No. 694977). 
[3] J. D. Comins, in Surface Brillouin Scattering, edited by A. Every and W. Sachse, Handbook of Elastic Properties of Solids, Liquids and Gases Vol. 1 (Academic Press, San Diego, 2001), pp. 349-378.

[4] E. Alonso-Redondo, M. Schmitt, Z. Urbach, C. Hui, R. Sainidou, P. Rembert, K. Matyjaszewski, M. Bockstaller, and G. Fytas, Nat. Commun. 6, 8309 (2015).

[5] B. Graczykowski, M. Sledzinska, F. Alzina, J. Gomis-Bresco, J. S. Reparaz, M. R. Wagner, and C. M. Sotomayor Torres, Phys. Rev. B 91, 075414 (2015).

[6] T. Still, W. Cheng, M. Retsch, R. Sainidou, J. Wang, U. Jonas, N. Stefanou, and G. Fytas, Phys. Rev. Lett. 100, 194301 (2008).

[7] F. Kargar, B. Debnath, J.-P. Kakko, A. Säynätjoki, H. Lipsanen, D. L. Nika, R. K. Lake, and A. A. Balandin, Nat. Commun. 7, 13400 (2016).

[8] B. F. Kennedy, P. Wijesinghe, and D. D. Sampson, Nat. Photon. 11, 215 (2017).

[9] B. Hillebrands, Rev. Sci. Instrum. 70, 1589 (1999).

[10] T. Sebastian, K. Schultheiss, B. Obry, B. Hillebrands, and H. Schultheiss, Front. Phys. 3, 35 (2015).

[11] J. Chan, A. H. Safavi-Naeini, J. T. Hill, S. Meenehan, and O. Painter, Appl. Phys. Lett. 101, 081115 (2012).

[12] A. H. Safavi-Naeini and O. Painter, Opt. Express 18, 14926 (2010).

[13] J.-C. Beugnot, S. Lebrun, G. Pauliat, H. Maillotte, V. Laude, and T. Sylvestre, Nat. Commun. 5, 5242 (2014).

[14] N. T. Otterstrom, R. O. Behunin, E. A. Kittlaus, Z. Wang, and P. T. Rakich, Science 360, 1113 (2018).

[15] K. R. Subbaswamy and A. A. Maradudin, Phys. Rev. B 18, 4181 (1978).

[16] V. Bortolani, F. Nizzoli, G. Santoro, and J. R. Sandercock, Phys. Rev. B 25, 3442 (1982).

[17] C. E. Bottani, G. Ghislotti, and P. Mutti, J. Phys.: Condens. Matter 6, L85 (1994).

[18] P. T. Rakich, C. Reinke, R. Camacho, P. Davids, and Z. Wang, Phys. Rev. X 2, 011008 (2012).

[19] C. Wolff, M. J. Steel, B. J. Eggleton, and C. G. Poulton, Phys. Rev. A 92, 013836 (2015).

[20] O. Florez, P. F. Jarschel, Y. A. V. Espinel, C. M. B. Cordeiro, T. P. Mayer Alegre, G. S. Wiederhecker, and P. Dainese, Nat. Commun. 7, 11759 (2016).

[21] H. Shin, W. Qiu, R. Jarecki, J. A. Cox, R. H. Olsson Iii, A. Starbuck, Z. Wang, and P. T. Rakich, Nat. Commun. 4, 1944 (2013).

[22] S. G. Johnson, M. Ibanescu, M. A. Skorobogatiy, O. Weisberg, J. D. Joannopoulos, and Y. Fink, Phys. Rev. E 65, 066611 (2002)

[23] Y. Pennec, V. Laude, N. Papanikolaou, B. Djafari-Rouhani, M. Oudich, J. S. El, J. C. Beugnot, J. M. Escalante, and A. Martínez, Nanophotonics 3, 413 (2014).
[24] J. A. Rogers and J.-H. Ahn, Silicon Nanomembranes: Fundamental Science and Applications (John Wiley \& Sons, New York, 2016).

[25] R. H. Olsson III and I. El-Kady, Meas. Sci. Technol. 20, 012002 (2009).

[26] B. Graczykowski, A. El Sachat, J. S. Reparaz, M. Sledzinska, M. R. Wagner, E. Chavez-Angel, Y. Wu, S. Volz, Y. Wu, F. Alzina, and C. M. Sotomayor Torres, Nat. Commun. 8, 415 (2017).

[27] M. Maldovan, Nat. Mater. 14, 667 (2015).

[28] M. Grimsditch, R. Bhadra, and I. K. Schuller, Phys. Rev. Lett. 58, 1216 (1987).

[29] R. Bhadra, M. Grimsditch, I. K. Schuller, and F. Nizzoli, Phys. Rev. B 39, 12456 (1989).

[30] J. Groenen, F. Poinsotte, A. Zwick, C. M. Sotomayor Torres, M. Prunnila, and J. Ahopelto, Phys. Rev. B 77, 045420 (2008).

[31] J. Cuffe, E. Chávez, A. Shchepetov, P.-O. Chapuis, E. H. El Boudouti, F. Alzina, T. Kehoe, J. Gomis-Bresco, D. Dudek, Y. Pennec, B. Djafari-Rouhani, M. Prunnila, J. Ahopelto, and C. M. Sotomayor Torres, Nano Lett. 12, 3569 (2012).

[32] X. Zhang, R. Sooryakumar, and K. Bussmann, Phys. Rev. B 68 , 115430 (2003).

[33] J. A. Forrest, K. Dalnoki-Veress, and J. R. Dutcher, Phys. Rev. E 58, 6109 (1998).

[34] V. Bortolani, A. Marvin, F. Nizzoli, and G. Santoro, J. Phys. C 16, 1757 (1983).

[35] B. D. Rouhani and E. M. Khourdifi, in Light Scattering in Semiconductor Structures and Superlattices, NATO ASI Series, edited by D. J. Lockwood and J. F. Young (Springer US, New York, 1991), pp. 139-158.

[36] I. E. Psarobas, N. Papanikolaou, N. Stefanou, B. DjafariRouhani, B. Bonello, and V. Laude, Phys. Rev. B 82, 174303 (2010).

[37] L. Dobrzynski, Surf. Sci. 180, 489 (1987).

[38] B. Djafari-Rouhani, S. El-Jallal, and Y. Pennec, C. R. Phys. 17, 555 (2016).

[39] L. Hounsome, R. Jones, M. Shaw, and P. Briddon, Phys. Status Solidi A 203, 3088 (2006).

[40] M. Grimsditch, E. Kisela, and M. Cardona, Phys. Status Solidi A 60, 135 (1980).

[41] J. J. Hall, Phys. Rev. 161, 756 (1967).

[42] See Supplemental Material at http://link.aps.org/supplemental/ 10.1103/PhysRevB.99.165431 for details of the finite element method model, BLS backscattering geometry, BLS spectrum of higher order SH modes, and simplified formulas for PE effect .

[43] O. L. Lazarenkova and A. A. Balandin, Superlattices Microstruct. 33, 95 (2003).

[44] J. He, B. Djafari-Rouhani, and J. Sapriel, Phys. Rev. B 37, 4086 (1988).

[45] E. L. Albuquerque, M. C. Oliveros, and D. R. Tilley, J. Phys. C 17, 1451 (1984). 